\title{
Using the etalon effect for in situ balancing of the Advanced Virgo arm cavities
}

\author{
S Hild ${ }^{1}$, A Freise ${ }^{1}$, M Mantovani ${ }^{3}$, S Chelkowski ${ }^{1}$, J Degallaix $^{2}$ \\ and R Schilling ${ }^{2}$ \\ ${ }^{1}$ School of Physics and Astronomy, University of Birmingham, Edgbaston, Birmingham B15 \\ 2TT, UK \\ ${ }^{2}$ Max-Planck-Institut für Gravitationsphysik (Albert-Einstein-Institut) and Leibniz Universität \\ Hannover, Callinstr. 38, D-30167 Hannover, Germany. \\ ${ }^{3}$ European Gravitational Observatory (EGO), Via E Amaldi, I-56021 Cascina (PI), Italy \\ E-mail: hild@star.sr.bham.ac.uk
}

Received 11 August 2008, in final form 11 November 2008

Published 22 December 2008

Online at stacks.iop.org/CQG/26/025005

\begin{abstract}
Several large-scale interferometric gravitational-wave detectors use resonant arm cavities to enhance the light power in the interferometer arms. These cavities are based on different optical designs: One design uses wedged input mirrors to create additional optical pick-off ports for deriving control signals. The second design employs input mirrors without wedge and thus offers the possibility of using the etalon effect inside the input mirrors for tuning the finesse of the arm cavities. In this paper, we introduce a concept of maximum flexibility that combines both of these options, by featuring wedges at the input mirrors and using the etalon effect instead in the end mirrors. We present a design for the arm cavities of Advanced Virgo. The paper focusses on evaluating the influence of etalon imperfections onto the overall Advanced Virgo performance. We use numerical simulations to derive requirements for the manufacturing accuracy of an end mirror etalon for Advanced Virgo. Furthermore, we give analytical approximations for the achievable tuning range of an imperfect etalon depending on the curvature and orientation mismatch of the two etalon surfaces. We evaluate the displacement noise originating from temperature driven optical phase noise of the etalon. In addition the influence of the etalon effect onto other Advanced Virgo subsystems such as the alignment sensing and control is analysed.
\end{abstract}

PACS numbers: $04.80 . \mathrm{Nn}$, 07.60.Ly, 95.75.Kk, 95.55.Ym

(Some figures in this article are in colour only in the electronic version) 


\section{Introduction}

The first generation of large-scale interferometric gravitational-wave detectors [1-4] has successfully been constructed. Simultaneous long-duration data takings have been performed. Currently, most of the detectors undergo minor upgrades including only a few hardware changes. However, in a few years major upgrades will be performed leading to the so-called second generation gravitational-wave detectors. The construction of these instruments, such as Advanced Virgo [6] and Advanced LIGO [5], will include major changes of almost all subsystems. In Advanced Virgo, amongst others, the beam geometry inside the arm cavities will be changed and new mirrors will be installed. This provides us with the possibility of introducing and evaluating new concepts for the optical layout of the arm cavities. As we will show, it is possible to optimize the arm cavity design by using wedged input mirrors and employing the etalon effect at the end mirrors in order to realize arm cavities with tunable finesse, i.e. adjustable losses.

We start in section 2 by giving a brief motivation for using arm cavities providing in situ balancing. In section 3, we give a brief description of the advantages and disadvantages of using either wedged or unwedged input mirrors, before we propose a concept which combines the advantages of both options. Analytical calculations and numerical simulations are used in section 4 to show how the tunable range is reduced by imperfections of the etalon, such as the curvature mismatch or nonparallelism of the two etalon surfaces. In section 5, we analyse the influence of imperfect optics onto other subsystems of Advanced Virgo, such as for instance, the auto-alignment system. A summary is given in section 6 .

\section{Motivation for arm cavities providing in situ balancing}

A Michelson interferometer with unbalanced arm cavities (arm cavities with differing finesse and/or losses) is undesirable for the following reasons:

- Due to the different light storage times inside two arm cavities, the common mode rejection for several technical noise sources is reduced resulting in a stronger noise coupling into the gravitational-wave channel $[13,14]$. In particular, the coupling of radiation pressure noise originating from laser intensity noise into the main interferometer output is increased.

- The dark fringe contrast is reduced resulting in an increased waste light level at the detection port and a reduced power recycling gain.

In practice, mismatches of the reflectivities, the cleanliness and the surface roughness of the mirrors limit the achievable balance of the two arm cavities. Since the mirrors of future gravitational-wave detectors are likely to be suspended by quasi -monolithic suspension (instead of metal wire loops) it will be risky to properly clean them after installation. Also exchanging a mirror will take much longer than in first generation instruments and would therefore cause increased downtime of the instrument.

Hence, it might be beneficial to utilize the etalon effect in one or both of the cavity end mirrors in order to balance the two arm cavities in situ. Balancing the interferometer arms might significantly reduce the technical problems listed above and therefore speed up the commissioning of the second generation instruments.

\section{Principle optical layout of the Advanced Virgo arm cavities}

\subsection{Etalon to tune the mirror reflectance}

The arm cavity configuration of initial Virgo is shown in figure $1(A)$. The end mirror is a wedged curved/flat mirror with an anti reflective (AR) coating at the back surface. The input 


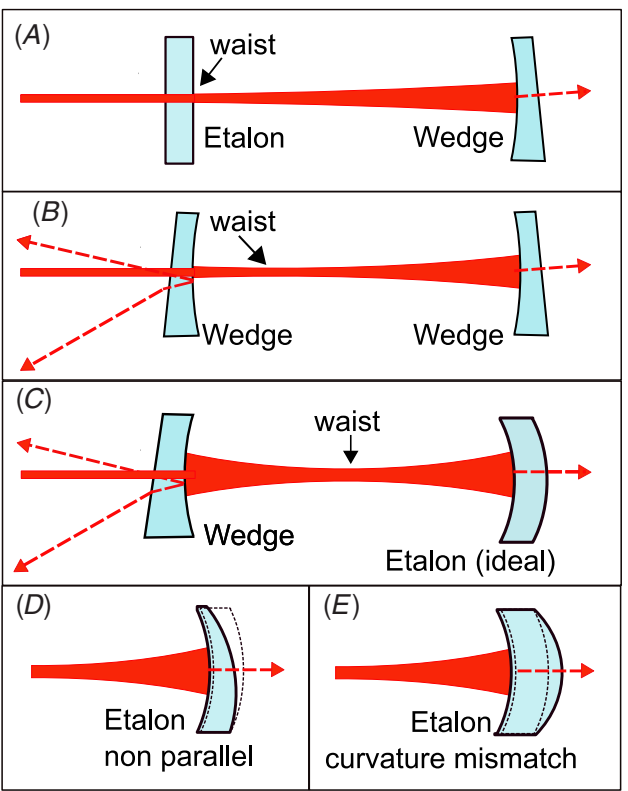

Figure 1. The plot shows various arm cavity configurations used by currently operating interferometers, as well as potential configurations for future detectors. From the left side the light enters the arm cavity formed by the input mirror (left) and end mirror (right). (A) Initial Virgo configuration: the input mirror is a flat/flat etalon, while the end mirror is curved. $(B)$ Initial LIGO configuration: in contrast to $(A)$ the input mirror features a wedge (and is slightly curved). $(C)$ Design of the Advanced Virgo arm cavities as proposed by the authors: both mirrors are identically curved in order to shift the beam waist to the centre of the cavity. The input mirror features a wedge, while the etalon effect of the end mirror is used. In order to maximize the etalon effect both surfaces of the end mirror have to be curved identically. $(D)$ and $(E)$ show a non-perfect etalon. The influence of both illustrated imperfections, non parallelism $(D)$ and curvature mismatch $(E)$ will be analysed in section 5 of this paper.

mirror is a plane/plane mirror (featuring no wedge) with an AR coating and the mirror coating on the left and right surfaces, respectively. Due to the etalon effect the overall reflectance of the input mirror changes depending on the microscopic tuning of the etalon, i.e. the optical path length inside the etalon substrate. The microscopic tuning of the etalon can be controlled by changing the substrate temperature of the etalon, by means of the temperature-dependent index of refraction.

\subsection{Wedges and pick-off beams}

For the arm cavities of initial LIGO a different approach was chosen. As indicated by figure $1(B)$ the input mirror is wedged, thus any potential etalon effect is suppressed, so that even without any temperature control of the input mirror the cavity finesse is stable. This concept is simple and robust, but lacks any possibility of balancing the arm cavities.

The main benefit of featuring wedged input mirrors is the availability of additional pick-off beams from the input mirror AR coating. These beams can be used for deriving further control signals. For advanced gravitational-wave detectors the availability of many control ports with different signal content seems highly desirable, especially because all second generation 
instruments will employ signal recycling [9], which strongly increases the complexity of the length sensing and control systems $[10,11]$.

\subsection{Proposed optical layout for arm cavities in advanced interferometers}

While the optical design for Advanced Virgo is not yet complete, it has been decided to shift the beam waist to the centre of the cavity. This will maximize the beam spot size on both mirrors thus reducing the effects of thermal noise from the mirror substrates and the dielectric coatings. We can summarize the requirements and boundary conditions for an optimal optical design of the arm cavities for the Advanced Virgo advanced detector as follows:

- Wedged input mirrors to create additional pick-off beams,

- Tunable cavity finesse (the tuning range of approximately a few percent),

- Identical radius of curvature of the input and end mirrors (beam waist centred in the arm cavity).

Figure $1(C)$ shows a schematic of the arm cavity concept that we propose. The input mirror is flat/curved featuring a wedge, thus two pick-off beams are created. Since no etalon effect is present, the overall reflectance of the input mirror is independent of the temperature inside the substrate. Having no etalon effect in the input mirror has the advantage that the requirements for the performance of a thermal compensation system [12] can be relaxed, i.e. only thermal lensing needs to be compensated for. The end mirror has no wedge $\mathrm{e}^{3}$ and serves as etalon. In order to provide a reasonable tuning range of the overall reflectance of the end mirror, its back surface carries a dielectric coating of moderate reflectance (tens of \%). The best etalon performance can be achieved by also curving the back surface of the etalon, ensuring perfect mode matching of the cavity beam into the etalon.

The performance of such an ideal etalon configuration is easily computable and, in principle, no additional problems should occur, assuming the optical path length, i.e. the temperature inside the etalon substrate is well controlled (see section 4.2). However, in reality we have to deal, at least to some extent, with a non-perfect etalon. Etalon imperfections originating from limited accuracy in the manufacturing process of the etalon lead to a nonperfect wavefront matching inside the etalon. In addition, light absorption at the front surface coating can induce thermal deformation and thermal lensing inside the etalon. Any imperfections of the etalon do not only reduce the available tuning range of the etalon reflectance, but also influence the light field inside the arm cavity. An analysis of potential distortion effects originating from a non-perfect etalon is carried out in the section 5.

\subsection{Comparison of input mirror etalon and end mirror etalon}

The performance of an arm cavity inside a laser-interferometric gravitational-wave detector is mainly determined by the finesse of the arm cavity, $F$, and the cavity reflectance, $R$, (seen from the beam-splitter). Both $F$ and $R$ depend, in different ways, on the same parameters, namely the transmissions of the two cavity mirrors and the losses inside the cavity. Two etalons are required to the simultaneous balance $F$ and $R$, one at the input mirror and one at the end mirror. The application of one etalon per cavity only allows us to the balance either $F$ or $R$.

State-of-the-art coating technology produces accurate coating refelectivities, very close to the design values. However, experience from commissioning of the currently operating laser-interferometric gravitational-wave detectors revealed the presence of unexpected losses inside the arm cavities, which are the object of intensive investigations. Therefore, the

3 At the end mirror no additional pick-off beams are required because the transmitted beam is already accessible. 
Table 1. Set of Advanced Virgo arm cavity parameters we use in this paper. Loss, $L_{i}$ and transmittance, $T_{i}$, are given in power, the respective amplitude reflectivities calculates as $\rho_{i}=\sqrt{1-T_{i}-L_{i}}$. The radii of curvature of the mirror surfaces are indicated by $R_{C i}$. (In the analytical description we assume mirrors of infinite size, while the FFT simulations use mirrors of $35 \mathrm{~cm}$ in diameter.)

\begin{tabular}{lll}
\hline Input mirror & $T_{1}$ & 0.007 \\
& $L_{1}$ & $50 \mathrm{ppm}$ \\
& $R_{C 1}$ & $1910 \mathrm{~m}$ \\
\hline End mirror front & $T_{2}$ & $50 \mathrm{ppm}$ \\
& $L_{2}$ & $50 \mathrm{ppm}$ \\
& $R_{C 2}$ & $1910 \mathrm{~m}$ \\
\hline End mirror back & $T_{3}$ & Variable $(0.8-0.98)$ \\
& $L_{3}$ & None \\
& $R_{C 3}$ & $1910 \mathrm{~m}($ ideal $)$ \\
\hline Cavity length & & $3000 \mathrm{~m}$ \\
Mirror diameter & & $0.35 \mathrm{~m}$ \\
\hline
\end{tabular}

authors believe that the main imbalancing of the Advanced Virgo arm cavities originates from differential losses. This favours a solution in which the etalons are placed at the end mirrors.

\section{Application of an ideal etalon in Advanced Virgo}

In this section, we apply the concept proposed in the previous section to the design of the Advanced Virgo detector. We use analytical calculations to describe the tuning range of the etalon in dependence of the reflectance of the etalon back surface. In addition, we analyse optical phase noise originating from temperature fluctuations inside the etalon. The optical parameters of Advanced Virgo, used throughout this paper, are displayed in table 1. Please note that we give transmittance, $T_{i}$, and loss, $L_{i}$, in power, while the reflectance, $\rho_{i}=\sqrt{1-T_{i}-L_{i}}$, is given in amplitude.

\subsection{Achievable tuning range of an ideal etalon}

The reflectance of a lossless etalon, $\rho_{E}$ can be written as

$$
\rho_{E}=\rho_{2}-\frac{\rho_{3}\left(1-\rho_{2}^{2}\right)}{\exp (-2 \mathrm{i} \phi)-\rho_{2} \rho_{3}},
$$

with $\rho_{2}$ and $\rho_{3}$ being the amplitude reflectance of the etalon front and back surfaces, respectively, and $\phi$ being the tuning of the etalon with $\phi=180^{\circ}$ referring to one free spectral range. The absolute value of $\rho_{E}$ can be used to determine the power reflectance or transmittance of the etalon. Figure 2 shows the overall transmittance of a cavity end mirror etalon using the parameters given in table 1 . The difference between the maximum and minimum transmittance can be called 'maximum compensation' and describes what amount of differential losses in two arm cavities can be compensated for by tuning the end mirror etalon.

The finesse, $F$, of the arm cavity can be computed using

$$
F=\frac{\pi}{2} \arcsin ^{-1}\left(\frac{1-\rho_{1} \rho_{E}}{2 \sqrt{\rho_{1} \rho_{E}}}\right) .
$$




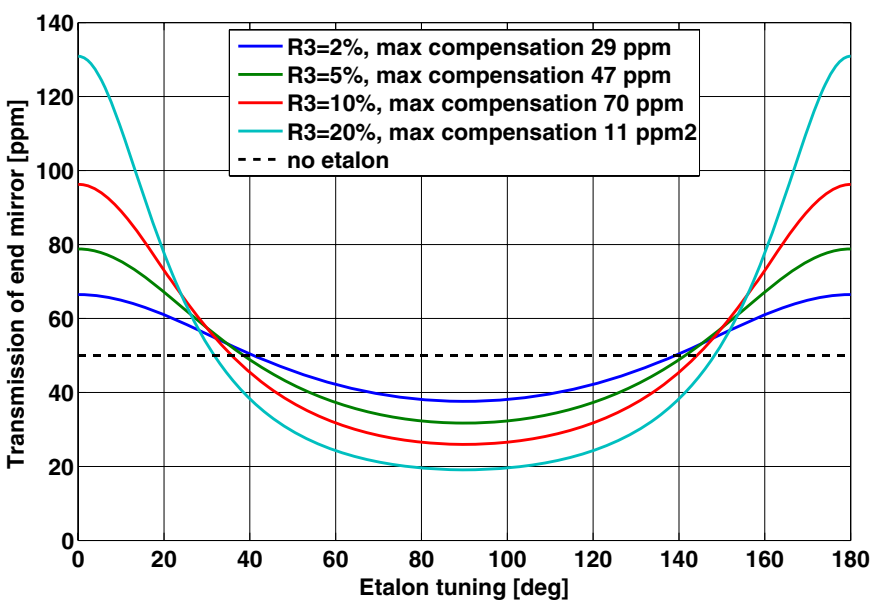

Figure 2. The plot shows the power transmittance of the end mirror using the etalon effect as a function of the tuning angle $\phi$. The etalon is assumed to have a power reflectance of $R_{2}=1-$ $50 \mathrm{ppm}$ at the front face and of $R_{3}$ at the back surface.

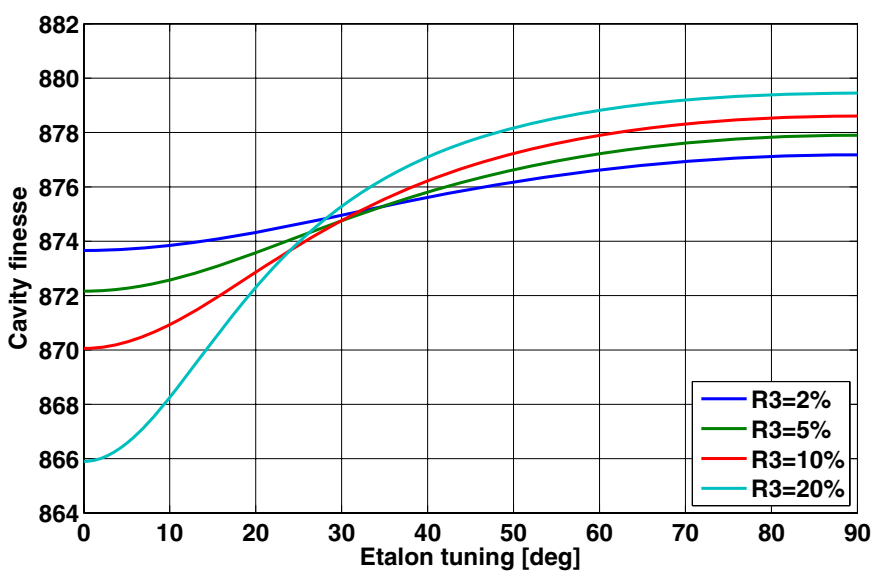

Figure 3. Arm cavity finesse in dependence of the etalon tuning and the power reflectance $R_{3}$ of the etalon back surface.

Figure 3 shows the finesse, $F$, of the cavity as a function of the etalon tuning using the mirror parameters shown in table 1 .

\subsection{Optical phase noise}

The overall reflectance of the etalon differs from that of an ordinary mirror not only in amplitude but also in phase. This can be interpreted such that the apparent position of the end mirror is influenced by the etalon tuning. This of course couples etalon fluctuations into optical phase noise, i.e. apparent displacement noise of the test masses at the end of the arm cavities. The apparent position change of the mirror can be computed as

$$
\Delta x_{E}=\frac{\lambda}{4 \pi} \arctan \left(\frac{\operatorname{Im}\left(\rho_{E}\right)}{\operatorname{Re}\left(\rho_{E}\right)}\right) .
$$




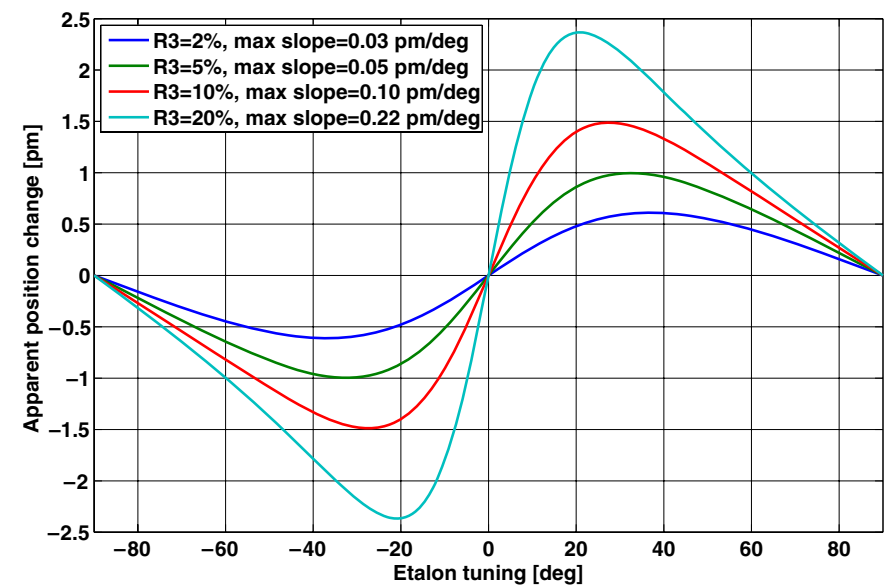

Figure 4. The plot shows the apparent position change of the end mirror as a function of the etalon tuning. The maximum slope around the zero tuning point is reported in the label.

Figure 4 shows the apparent position change as a function of the etalon tuning for a few examples. For a 'worst case scenario' of an etalon back surface coating of a power reflectance of $R_{3}=0.2$ and an etalon operating point with the strongest slope, the optical phase noise amounts to $n_{p}\left(\mathrm{pm} \mathrm{deg}^{-1}\right)=0.22 \mathrm{pm} \mathrm{deg}^{-1}$. While in figure 4 the etalon tuning is given in units of degree, it can also be expressed in units of length, using $1^{\circ} \hat{=} \lambda / 360$, where $\lambda=1064 \mathrm{~nm}$ is the wave length of the laser light. This allows us to rewrite the optical phase noise as $n_{p}^{\prime}\left(\mathrm{m} \mathrm{m}^{-1}\right)=7.4 \times 10^{-5}$, which can be used to calculate the corresponding displacement noise spectrum, $\tilde{x}_{E}$, in dependence of the amplitude spectral density of the effective temperature fluctuations, $\tilde{T}_{E}(f)$, inside the etalon:

$$
\tilde{x}_{E}(f)=\tilde{T}_{E}(f) \cdot \frac{\mathrm{d} n}{\mathrm{~d} T} \cdot l_{E} \cdot n_{p}^{\prime}
$$

where $l_{E}$ is the thickness of the etalon, and $\mathrm{d} n / \mathrm{d} T$ is the change of the index of refraction of the etalon substrate with temperature. As shown in [16] the effective temperature fluctuations of the etalon have to be kept below $10^{-10} \mathrm{~K} / \sqrt{\mathrm{Hz}}$ for most frequencies within the detection band in order to avoid spoiling the Advanced Virgo sensitivity. We can compare this requirement to the actually present temperature fluctuations, assuming that these are (for frequencies in the detection band) entirely driven by Brownian fluctuations. Using equation (5.2) from [15] we can describe the effective temperature fluctuations of the etalon to be

$$
\tilde{T}_{\mathrm{E}}(f)=\sqrt{\frac{4 k_{b} T^{2} \kappa}{(\rho C)^{2} l_{\text {eta }}} \frac{1}{\pi R_{b}^{4}(2 \pi f)^{2}}}
$$

where $k_{b}$ is the Boltzmann constant, $T$ is the temperature of the etalon, $\kappa$ is the thermal conductivity, $\rho$ is the density of the etalon, $C$ is the heat capacity, and $R_{b}$ is the radius of the laser beam inside the etalon substrate. It turns out that the Brownian temperature fluctuations are at least four orders of magnitude below the temperature requirement of the etalon [16]. 


\section{Analysis of etalon imperfections}

So far we assumed a geometrically perfect etalon with parallel surfaces. In practice, the etalon substrates will deviate from a perfect shape. The geometrical deviations with the largest impact on the reflected light are

- deviation of parallelism (i.e. relative misalignment)

- mismatch in spherical curvature.

The first case is illustrated in figure 1(D). The two etalon surfaces have identical curvature, but are misaligned in respect to each other, i.e. not parallel. Figure $1(E)$ shows the case of curvature mismatch of the two etalon surfaces.

In order to evaluate the effects of potential etalon imperfections on the arm cavity performance for Advanced Virgo we use two different methods: method 1 makes use of Hermite-Gauss coupling coefficients (HGCC). For method 2 we use FFT-based numerical simulations. In addition, we have used the interferometer simulation software FINESSE [20, 21] to cross-check the results of the other two methods. The results from FINESSE agreed well with the other two methods, and are hence omitted from the plots in this section for clarity.

A short description of each of the two methods is given in the sections 5.1 and 5.2. We use both methods to calculate how the achievable tuning range of an etalon is influenced by its imperfections (section 5.3). Finally, using the example of the alignment sensing and control system, we evaluate how the etalon imperfections can potentially influence other subsystems of the Advanced Virgo detector (section 5.4).

\subsection{Method 1: Hermite-Gauss coupling coefficients}

In this section, we compute coupling coefficients which determine how much light from a $\mathrm{TEM}_{00}$ mode incident on the etalon will be scattered into higher-order modes due to the imperfection of the etalon back surface. In a first approximation, we consider the light scattered into higher-order modes as additional losses. This is an approximation because in reality the coupling into higher-order modes can be enhanced or reduced by the arm cavity resonance. However, as we show in the following, analytic expressions derived with this method match the results from numerical simulations very well.

A beam profile of a fundamental Gaussian beam (for a beam with a given wavelength $\lambda$ ) can be completely determined by two parameters: the minimum spot size $w_{0}$ (called beam waist) and the position $z$ of the beam waist along the $z$-axis. A convenient method of expression of these two parameters is the Gaussian beam parameter,

$$
q(z)=\mathrm{i} z_{\mathrm{R}}+z=q_{0}+z,
$$

with the Rayleigh range $z_{R}$ defined as

$$
z_{\mathrm{R}}=\frac{\pi w_{0}^{2}}{\lambda}
$$

In the following, we describe the light field in the arm cavity and the etalon using HermiteGauss modes which are an orthonormal set of functions which can be used to describe deviations of the beam from its fundamental Gaussian shape. The scalar electrical field describing a beam propagating along the $\mathrm{z}$-axis can then be written as

$$
E(x, y, z)=E_{0} \sum_{n m} c_{n m} u_{n m}(x, y, z) \exp (\mathrm{i} \omega t-k z)
$$


in which the set of Hermite-Gauss modes $u_{n m}$ is defined by the Gaussian beam parameter $q$ of the beam, and the coefficients $c_{n m}$ determine the mode composition of $E$. We assume that the arm cavity and the incoming light are perfectly matched, and the light is in a zero-order mode described by $q_{c}$ being the beam parameter of the cavity eigenmode.

When a beam is passing through or reflected by a spherical surface a beam parameter $q_{1}$ is transformed to $q_{2}$. The transformation of the beam parameter can be performed by the $\mathrm{ABCD}$ matrix formalism [8]. In our case the beam reaching the end mirror passes first into the substrate, which causes the beam parameter $q_{c}$ to be transformed as

$$
q_{t}=n \frac{q_{c}}{\frac{n-1}{R_{C}} q_{c}+1}
$$

with $R_{\mathrm{C}}$ the radius of curvature of that surface and $n$ the index of refraction of the substrate. Then upon reflection from the back surface ${ }^{4}$ we get a second transformation

$$
-q_{r}^{*}=\frac{q_{t}}{\frac{-2}{R_{C}} q_{t}+1}
$$

with the ${ }^{*}$ indicating the complex conjugate. In general $q_{t}$ and $q_{r}$ will be different, i.e. the incoming and reflected beam will have a different shape. In order to compute how much of the light will remain in the cavity eigenmode we have to describe the reflected 00 mode, $u_{00}\left(q_{r}\right)$, in the base system of $u_{n m}\left(q_{t}\right)$. Such a base change can be described by the coupling coefficients $k_{n m n^{\prime} m^{\prime}}$ :

$$
u_{n m}\left(q_{t}\right)=\sum_{n^{\prime} m^{\prime}} k_{n m n^{\prime} m^{\prime}} u_{n^{\prime} m^{\prime}}\left(q_{r}\right) .
$$

We are interested in how much of the reflected light is coupled back into the fundamental cavity eigenmode. The respective coupling coefficient $k_{0000}\left(q_{1}, q_{2}\right)$ can be found in the literature; for example, in [7] the coupling coefficients are defined using the mode-mismatch parameters $K_{0}$ and $K$ given as

$$
K=\frac{1}{2}\left(K_{0}+\mathrm{i} K_{2}\right),
$$

where $K_{0}=\left(z_{\mathrm{R}, 1}-z_{\mathrm{R}, 2}\right) / z_{\mathrm{R}, 2}$ and $K_{2}=\left(z_{1}-z_{2}\right) / z_{\mathrm{R}, 2}$. This can be also written as

$$
K=\frac{\mathrm{i}\left(q_{1}-q_{2}\right)^{*}}{2 \operatorname{Im}\left(q_{2}\right)}
$$

This parameter is a measure of the mode mismatch between two $\mathrm{TEM}_{00}$ shapes defined by the Gaussian beam parameters $q_{1}$ and $q_{2}$.

If we assume perfect alignment of the front and back surfaces, but allow different radii of curvature of the two etalon surfaces, the coupling coefficient of interest is then given as

$$
k_{0000}\left(q_{1}=q_{r}, q_{2}=q_{t}\right)=\frac{\sqrt{1+K_{0}}}{1+K^{*}} .
$$

Then the effect of the etalon can be computed as before by replacing $\rho_{3}$ by $\left|k_{0000}\right| \rho_{3}$. If on the other hand the surfaces have matched curvatures but the back surface is misaligned by an angle $\gamma$ the coefficient is given as

$$
k_{0000}\left(q_{r}, \gamma\right)=\exp \left(-\frac{\pi\left|q_{r}\right|^{2} \sin ^{2}(2 \gamma)}{2 \lambda \operatorname{Im}\left(q_{r}\right)}\right) .
$$

\footnotetext{
4 The short propagation through the mirror substrate can be neglected in this case.
} 


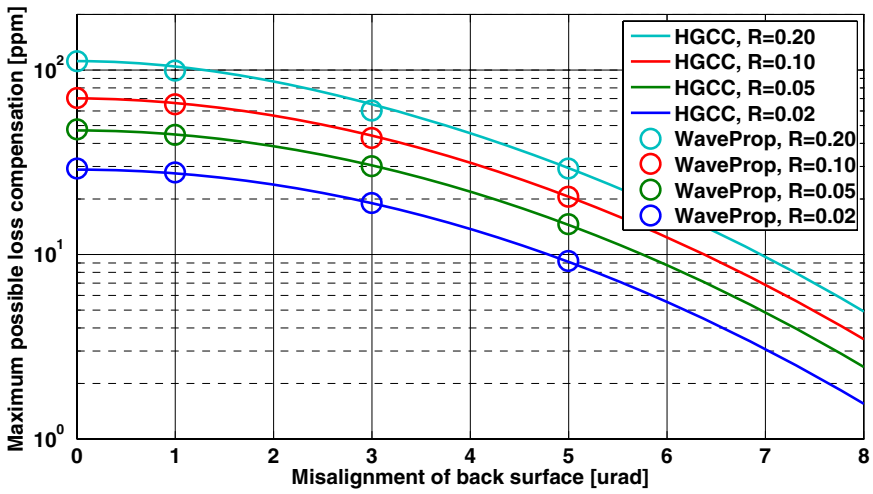

Figure 5. The plot shows the maximum loss that can be compensated for by the etalon, as a function of the etalon rear surface misalignment. The solid traces are computed from HermiteGauss coupling coefficients (HGCC), while the data points represented by a circle are derived from WaveProp simulations.

\subsection{Method 2: FFT-based simulations}

The second method we used to evaluate the influence of imperfections onto the etalon performance is based on FFT simulations using the program WaveProp. WaveProp is a tool for simulating the propagation of electromagnetic waves, using discrete Fourier transformation. The program is available at [17]. The method of FFT propagation is described, e.g., by Siegmann [8] and by Vinet et al [19].

\subsection{Tuning range of an imperfect etalon}

Using the two methods that have been laid out in the previous sections, we can now calculate how much the etalon performance is reduced depending on its geometrical imperfections. In general, these geometrical imperfections reduce the wavefront overlap inside the etalon and therefore reduce the achievable tuning range of the etalon reflectivity. Figure 5 shows the maximum loss compensation possible with the parameters of an Advanced Virgo arm cavity (see table 1). For different reflectances of the etalon rear surface we plotted the achievable loss compensation over the misalignment of the etalon rear surface. The maximum possible loss compensation strongly depends on the misalignment of the etalon rear surface. Already for a misalignment of 1 urad the compensation range is reduced by about $10 \%$ and roughly halved for a misalignment of 3.5 urad.

Figure 6 shows the maximum loss that can be compensated for as a function of the curvature of the etalon back surface. At $R_{C}=1910$ the curvature is matched to the beam and values for compensation are maximized. However, even with moderate deviations from a matched curvature the range of the etalon is reduced only very little. A deviation of the rear surface radius of curvature of about $100 \mathrm{~m}$ reduces the maximum compensation range by only a few percent.

In summary, the tuning range of the etalon and therefore also the maximal achievable loss compensation range strongly depends on the misalignment (non-parallelism) of the etalon rear surface, while the radius of curvature of the rear surface is rather uncritical. Therefore, it will be necessary to set tight parallelism requirements for the polishing accuracy of the etalon substrate. 


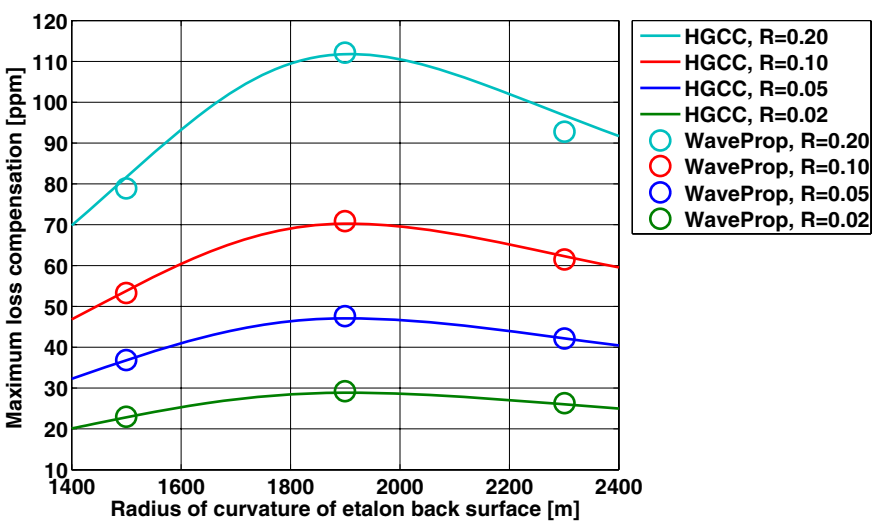

Figure 6. The plot shows the maximum differential loss that can be compensated for by the etalon as a function of the radius of curvature of the spherical back surface of the etalon. The solid traces are computed from Hermite-Gauss coupling coefficients (HGCC), while the data points represented by a circle are derived from WaveProp simulations. The results from both method show that the system is uncritical with respect to moderate deviations in curvature.

In case it turns out that with state-of-the-art technology it is impossible to achieve a misalignment of the rear surface of less than $1 \mathrm{urad}$, one might consider first measuring the non-parallelism of the polished etalon substrate and afterwards deciding on the actual reflectance of the rear coating, necessary to achieve the actual required loss compensation range.

\subsection{Simulation: influence of etalon effect onto alignment signals}

Imperfections of the etalon do not only decrease the achievable loss compensation, but may also harm other detector subsystems due to the additional creation of higher-order optical modes. Since the strongest disturbances are expected to show up in the global alignment sensing and control system of Advanced Virgo, we performed some detailed FINESSE simulations of the alignment control signals using an imperfect etalon.

The effect of a possible mismatch between the alignment of the back and front faces of the etalon was found to have very small influence onto the alignment error signals of the arm cavity. The coupling of etalon rear surfaces misalignment into the arm cavity alignment signals is about four to five orders of magnitude below the coupling of the etalon front surface misalignment [18]. Also the amount of first-order modes $\left(\mathrm{TEM}_{01}\right.$ and $\left.\mathrm{TEM}_{10}\right)$ originating from etalon imperfections is found to be negligible [18]. Thus from the point of view of the alignment control system there are no evident disadvantages of using an etalon as the end mirror of the Advanced Virgo arm cavities.

\section{Summary and conclusion}

We have presented a new arm cavity configuration for advanced gravitational-wave detectors. Our concept combines the advantages of the different currently used arm cavity designs and offers maximized flexibility, by providing access to all important interferometer ports and simultaneously allowing in situ balancing of the two arm cavities. Detailed calculations and numerical simulations have been performed to evaluate the performance 
of the proposed arm cavity design. These analyses considered ideal optical elements as well as the case of optics with significant imperfections. Our concept is found to have only negligible implications for the other detector subsystems. Due to the flexibility offered by arm cavities featuring the possibility of in situ tuning of the losses, the authors believe that this concept might speed up commissioning of the advanced gravitational-wave detectors.

\section{Acknowledgment}

We would like to thank Francios Bondu, Harald Lück, Guido Müller, Michele Punturo, Giovanni Losurdo and Raffaele Flaminio for fruitful discussions. This work has been supported by the Science and Technology Facilities Council (STFC), Max-Planck Society (MPG) and the European Gravitational Observatory (EGO). This document has been assigned LIGO Laboratory document number LIGO-P080078-00-Z.

\section{References}

[1] Hild S et al 2006 The status of GEO 600 Class. Quantum Grav. 23 S643-51

[2] Acernese F et al 2004 Status of VIRGO Class. Quantum Grav. 21 S385-94

[3] Sigg D et al 2004 Commissioning of LIGO detectors Class. Quantum Grav. 21 S409-15

[4] Takahashi R and the TAMA Collaboration 2004 Status of TAMA300 Class. Quantum Grav. 21 S403-8

[5] http://www.ligo.caltech.edu/advLIGO/

[6] http://wwwcascina.virgo.infn.it/advirgo/

[7] Bayer-Helms F 1984 Coupling coefficients of an incident wave and the modes of a spherical optical resonator in the case of mismatching Appl. Opt. 23 1369-80

[8] Siegman A E 1986 Lasers (Mill Valley: University Science Books) see also the errata at http://www-ee.stanford. edu/ siegman/lasers_book_errata.pdf

[9] Meers B J 1988 Recycling in laser-interferometric gravitational-wave detectors Phys. Rev. D 382317

[10] Grote H et al 2004 Dual recycling for GEO 600 Class. Quantum Grav. 21 S473-80

[11] Miyakawa O et al 2006 Measurement of optical response of a detuned resonant sideband extraction gravitational wave detector Phys. Rev. D 74022001

[12] Lawrence R et al 2002 Adaptive thermal compensation of test masses in advanced LIGO Class. Quantum Grav. 19 1803-12

[13] Somiya K et al 2006 Frequency noise and intensity noise of next-generation gravitational-wave detectors with RF/DC readout schemes Phys. Rev. D 73122005

[14] Somiya K et al 2007 Frequency noise and intensity noise of next-generation gravitational-wave detectors with RF/DC readout schemes Phys. Rev. D 75049905 (erratum)

[15] Braginski V B and Vyatchanin S P 2004 Corner reflectors and quantum-non-demolition measurements in gravitational wave antennae Phys. Lett. A 324 345-60

[16] Hild S and Freise A Advanced Virgo design: Optical phase noise originating from the etalon effect driven by thermo-refractive noise, Virgo note VIR-058A-08, available at https://pub3.ego-gw.it/codifier/

[17] Available at http://www.rzg.mpg.de/ros/WaveProp/

[18] Mantovani M et al The consequences of using the etalon effect to tune the arm cavity finesse on the alignment signals for Advanced Virgo, Virgo note VIR-027A-08, available at https://pub3.ego-gw.it/codifier/

[19] Vinet J-Y, Hello P, Man C N and Brillet A 1992 J. Phys. I (Paris) 21287

[20] Interferometer simulation software FINESEE, available at http://www.rzg.mpg.de/adf/

[21] Freise A et al 2004 Frequency-domain interferometer simulation with higher-order spatial modes Class. Quantum Grav. 21 S1067-74 